\title{
Interference with circRNA DOCK1 inhibits hepatocellular carcinoma cell proliferation, invasion and migration by regulating the miR-654-5p/SMAD2 axis
}

\author{
YUJUAN LU ${ }^{1}$, JINGZHI ZHANG ${ }^{2}$ and YANHUI WU ${ }^{3}$ \\ ${ }^{1}$ Department of Infectious Diseases, Zibo Central Hospital, Zibo, Shandong 255036; ${ }^{2}$ Department of Critical Care Medicine, \\ Zibo Integrated Chinese and Western Medicine Hospital, Zibo, Shandong 255026; ${ }^{3}$ Hepatic Surgery Center, Tongji Hospital, \\ Tongji Medical College, Huazhong University of Science and Technology, Wuhan, Hubei 430030, P.R. China
}

Received November 4, 2020; Accepted February 22, 2021

DOI: $10.3892 / \mathrm{mmr} .2021 .12247$

\begin{abstract}
Hepatocellular carcinoma (HCC) is the fourth most common cause of cancer-related death worldwide. The aim of the present study was to discuss the role of circular RNA (circRNA) dedicator of cytokinesis 1 (DOCK1) in HCC and whether it can affect cell proliferation, invasion and migration by regulating the microRNA (miR)-654-5p/SMAD2 axis. The expression levels of circRNA DOCK1, miR-654-5p and SMAD2 mRNA in HCC cells and transfected Hep3b cells were detected by reverse transcription-quantitative PCR analysis. SMAD2 protein expression levels in HCC cells and transfected Hep3b cells were analyzed by western blot analysis. The viability, proliferation, migration and invasion of transfected Hep3b cells was in turn detected by Cell Counting Kit-8, clone formation, wound healing and Transwell assays. The interaction of circRNA DOCK1 and miR-654-5p, miR-654-5p and SMAD2 was confirmed by the dual-luciferase reporter assay. As a result, the expression of circRNA DOCK1 and SMAD2 was increased, and miR-654-5p was decreased in HCC cells. circRNA DOCK1 directly targeted to miR-654-5p and miR-654-5p directly targeted to SMAD2. Interference with circRNA DOCK1 inhibited the proliferation, invasion and migration of HCC cells by upregulating miR-654-5p expression. The effects of circRNA DOCK1 knockdown could be partially reversed by transfection with a miR-654-5p inhibitor and SMAD2 overexpression. In conclusion, interference with circRNA DOCK1 inhibited proliferation, invasion and migration of HCC cells by regulating the miR-654-5p/SMAD2 axis.
\end{abstract}

Correspondence to: Dr Yanhui Wu, Hepatic Surgery Center, Tongji Hospital, Tongji Medical College, Huazhong University of Science and Technology, 1095 Jiefang Avenue, Wuhan, Hubei 430030, P.R. China

E-mail: yanhaiyhw@163.com

Key words: circular RNA dedicator of cytokinesis 1, proliferation, invasion and migration, microRNA-654-5p/SMAD2 axis, hepatocellular carcinoma

\section{Introduction}

Hepatocellular carcinoma (HCC) is a common digestive tract malignant tumor with poor prognosis in China, and is the fourth most common cause of cancer-related mortality worldwide, accounting for $7 \%$ of all cancer-related deaths $(1,2)$. The primary treatment strategy used for HCC is hepatectomy. However, $>80 \%$ of HCC cases are diagnosed at an advanced stage $(3,4)$, only $<5 \%$ of cases are candidates for surgical resection and the recurrence rate is $>70 \%$ within 5 years after surgery (5). At present, there is a lack of effective treatments for advanced HCC, particularly for patients following failure of sorafenib treatment $(6,7)$. Therefore, the design of targeted therapies for HCC has been a research hotspot.

Circular RNAs (circRNAs) are a class of endogenous non-coding RNAs that are produced by an exon and/or intron sequence of the original transcription by reverse splicing (8). Unlike classical linear RNAs with $5^{\prime}$ and $3^{\prime}$ ends, circRNAs produce covalent closed-loop constructs that prevent degradation by RNA exonuclease or RNase R, which results in increased stability of circRNAs compared with that of linear mRNAs (9). The expression of circRNAs in humans is tissue-specific, and various circRNAs have been reported to play important roles in HCC (10). A previous study demonstrated that circRNA dedicator of cytokinesis 1 (DOCK1) is highly expressed in thyroid cancer tissues (11). circRNA DOCK1 inhibits the expression of microRNA (miRNA/miR)-124 in thyroid cancer cells, blocks the signal transduction of the Janus kinase/signal transducer and activator of transcription/adenosine 5'-monophosphate-activated protein kinase signaling pathway and participates in the occurrence of thyroid cancer via downregulating miR-124 (11). circRNA DOCK1 has been reported to promote the progression of bladder cancer by regulating the circDOCK1/hsa-miR-132-3p/Sox 5 signaling pathway (12). Moreover, circRNA DOCK1 can inhibit miR-196a-5p-induced apoptosis by targeting baculoviral IAP repeat containing 3 in oral squamous cell carcinoma (13). However, to the best of our knowledge, the role of circRNA DOCK1 in HCC has not been previously reported.

It was previously reported that hsa-circ-u0085131 upregulates autophagy-related 7 via sponge adsorption to enhance 
the resistance of non-small cell lung cancer cells to cisplatin, resulting in autophagy (14). miR-654-5p downregulation has been observed in colorectal cancer cells, and miR-654-5p overexpression can inhibit colorectal cancer cell proliferation, invasion and migration by targeting hematopoietic lineage cell-specific protein (HCLS1)-associated protein X-1 (15). miR-654-5p has been reported to inhibit the occurrence of ovarian cancer through regulating the MYC, WNT and AKT signaling pathways (16). Furthermore, miR-654-5p downregulation can lead to the upregulation of transmembrane protein 52B in gastric cancer, which was found to promote cell invasion and metastasis both in vivo and in vitro (17). Previous studies have also demonstrated that SMAD2 is associated with the regression of HCC, whereas inhibition of SMAD2 can suppress the regression of HCC (18-20).

Therefore, the present study aimed to investigate the role of circRNA DOCK1 in HCC and determine whether it affects cell proliferation, invasion and migration by regulating the miR-654-5p/SMAD2 axis.

\section{Materials and methods}

Cell culture. A human normal hepatocyte cell line (HHL-5) and HCC cell lines (MHCC97-H, SK-Hep-1, Huh-7 and Hep3b) were purchased from BioVector NTCC, Inc. HHL-5 and HCC cells were cultured in Dulbecco's modified Eagle's medium (DMEM; Thermo Fisher Scientific, Inc.) supplemented with $10 \%$ fetal bovine serum (FBS; Thermo Fisher Scientific, Inc.), $100 \mathrm{U} / \mathrm{ml}$ penicillin and $100 \mathrm{U} / \mathrm{ml}$ streptomycin at $37^{\circ} \mathrm{C}$ with $5 \% \mathrm{CO}_{2}$ and saturated humidity. After resuscitation, cells of the 4 to 10th generation were used for subsequent experiments.

Plasmids and cell transfection. For the stable overexpression of circ-SMAD2, sequences were constructed into pcDNA3.1 (Invitrogen; Thermo Fisher Scientific, Inc.). The plasmids of short hairpin RNA (shRNA)-negative control (NC) and shRNA-DOCK1\#1/2 were commercially synthesized by Shanghai GenePharma Co., Ltd. Other plasmids were obtained from Guangzhou RiboBio Co., Ltd., as follows: mimic-NC (cat. no. miR1N0000001-1-5), miR-654-5p mimic (cat. no.miR10003330-1-5),NCinhibitor(cat.no.miR2N0000001-1-5) and miR-654-5p inhibitor (cat. no. miR20003330-1-5).

Hep3b cells in the logarithmic growth phase were selected and inoculated $\left(4 \times 10^{5}\right.$ cells) in a $6-\mathrm{cm}$ culture dish. Following culture for $24 \mathrm{~h}$, Hep3b cells were transfected with shRNA-NC $(50 \mathrm{nM})$, shRNA-DOCK1\#1/2 (50 nM), mimic-NC (50 nM), miR-654-5p mimic (50 nM), NC inhibitor (50 nM), miR-654-5p inhibitor (50 nM), pcDNA3.1-NC (50 nM) or pcDNA3.1-SMAD2 $(50 \mathrm{nM})$, or co-transfected with shRNA-DOCK1 + NC inhibitor, shRNA-DOCK1 + miR-654-5p inhibitor, shRNA-DOCK1 + pcDNA3.1-NC or shRNA-DOCK1 + pcDNA3.1-SMAD2 using Lipofectamine ${ }^{\circledR} 2000$ (Invitrogen; Thermo Fisher Scientific, Inc.) according to the manufacturer's protocol at $37^{\circ} \mathrm{C}$ for $24 \mathrm{~h}$. Hep3b cells without any treatment were used as the blank control group. At $24 \mathrm{~h}$ post-transfection, subsequent experiments were performed.

Reverse transcription-quantitative PCR (RT-qPCR). A PARIS $^{\text {TM }}$ kit (Thermo Fisher Scientific, Inc.) was used according to the manufacturer's protocols for cell fractionation. Briefly,
Hep3b cells $\left(1 \times 10^{5}\right)$ were mixed with $1 \mathrm{ml}$ cell fractionation buffer and centrifuged at $800 \mathrm{x}$ g for $20 \mathrm{~min}$ at room temperature. Subsequently, TRIzol ${ }^{\circledR}$ and TRIzol LS reagents (Invitrogen; Thermo Fisher Scientific, Inc.) were independently used to obtain total RNA from the nuclear pellet and cell supernatant. Total RNA was reverse-transcribed into cDNA using a cDNA reverse transcription kit (Roche Diagnostics). Subsequently, qPCR was performed using the SYBR Premix Ex Taq kit (Beyotime Institute of Biotechnology) and an FTC-3000P real-time PCR system (Shanghai Fengling Biotechnology Co., Ltd.). The thermocycling conditions were as follows: Initial denaturation at $95^{\circ} \mathrm{C}$ for $20 \mathrm{sec}$; followed by 40 cycles of denaturation at $95^{\circ} \mathrm{C}$ for $15 \mathrm{sec}$, annealing at $60^{\circ} \mathrm{C}$ for $30 \mathrm{sec}$ and extension at $72^{\circ} \mathrm{C}$ for $25 \mathrm{sec}$. The primer sequences were as follows: circRNA DOCK1 forward, 5'-CCTAGACGCGGAG TTTCCTG-3' and reverse, 5'-CCGCTCCTCTGGCATCA TAG-3'; SMAD2 forward, 5'-ATGTCGTCCATCTTGCCA TTC-3' and reverse, 5'-AACCGTCCTGTTTTCTTTAGCTT-3'; GAPDH forward, 5'-CATGAGAAGTATGACAACAGCCT-3' and reverse, 5'-AGTCCTTCCACGATACCAAAGT-3'; miR-654-5p forward, 5'-AGTGGAAAGATGGTGGGCCG-3' and reverse, 5'-GCTTCTAAAGGTGATGGTCAGCAG-3'; and U6 forward, 5'-CGCTTCACGAATTTGCGT-3' and reverse, 5'-CTCGCTTCGCAGCACA-3'. U6 and GAPDH were used as internal references for circRNA DOCK1, miR-654-5p and SMAD2, respectively. The expression levels were analyzed using the $2^{-\Delta \Delta \mathrm{Cq}}$ method (21).

Cell Counting Kit-8 (CCK-8) assay. Hep3b cells of each group were seeded (100 $\mu \mathrm{l}$ cell suspension/well; $2 \times 10^{3}$ cells) into 96-well plates. Following culture for 24,48 or $72 \mathrm{~h}$, cells were analyzed using a CCK-8 kit (Beyotime Institute of Biotechnology) according to the manufacturer's protocol. Absorbance was measured at a wavelength of $450 \mathrm{~nm}$ using a Thermomax microplate reader (Thermo Fisher Scientific, Inc.).

Colony formation assay. Hep3b cells of each group were seeded ( $1 \mathrm{ml}$ cell suspension/well; $5 \times 10^{2}$ cells) into 6 -well plates with DMEM supplemented with $10 \%$ FBS. Following culture for 14 days, cells were fixed with $4 \%$ formaldehyde at room temperature for $20 \mathrm{~min}$ and stained with $0.1 \%$ crystal violet solution at room temperature for $20 \mathrm{~min}$. The number of colonies ( $>50$ cells of each colony) was counted using a light microscope (magnification, x100; Olympus Corporation).

Wound healing assay. Hep3b cells of each group were seeded into 6 -well plates $\left(5 \times 10^{4}\right.$ cells/well $)$ and incubated in serum-free DMEM at $37^{\circ} \mathrm{C}$ with $5 \% \mathrm{CO}_{2}$. At $90 \%$ confluence, a 200- $\mu 1$ sterile pipette tip was used to scratch the cell monolayer. Cells were washed twice with PBS to remove cell debris. The wound was observed at 0 and $24 \mathrm{~h}$ using a light microscope (magnification, x100; Olympus Corporation). Five fields were used for quantification.

Transwell assay. The Transwell inserts were precoated with Matrigel at $37^{\circ} \mathrm{C}$ for $30 \mathrm{~min}$ (Becton-Dickinson and Company). Subsequently, $200 \mu \mathrm{l}$ serum-free DMEM containing $1 \times 10^{5}$ Hep3b cells was plated into the upper chamber. Then, $500 \mu 1$ DMEM supplemented with $10 \%$ FBS was plated into the lower chamber. Following incubation for $24 \mathrm{~h}$ at $37^{\circ} \mathrm{C}$ with $5 \% \mathrm{CO}_{2}$, 
cells were fixed with $4 \%$ paraformaldehyde for $30 \mathrm{~min}$ at room temperature and stained with hematoxylin for $30 \mathrm{~min}$ at room temperature. Invading cells were observed using a light microscope (magnification, x100; Olympus Corporation). Five fields were used for quantification.

Western blotting. Total protein was extracted from cells $\left(8 \times 10^{5}\right)$ using RIPA reagent (Beyotime Institute of Biotechnology). Protein concentration was determined by a BCA assay. Proteins $(30 \mu \mathrm{g})$ were separated via $10 \%$ SDS-PAGE, and separated proteins were subsequently transferred to PVDF membranes. Following blocking with $5 \%$ skimmed milk at room temperature for $2 \mathrm{~h}$, the membranes were incubated at $4^{\circ} \mathrm{C}$ for $12 \mathrm{~h}$ with primary antibodies targeted against SMAD2 (cat. no. ab40855; 1:2,000; Abcam) and GAPDH (cat. no. ab9485; 1:1,000; Abcam). Following washing three times with TBS with $0.05 \%$ Tween-20, the membranes were incubated with a HRP-conjugated goat anti-rabbit secondary antibody (cat. no. ab6721; $1: 2,000$; Abcam) at $37^{\circ} \mathrm{C}$ for $1 \mathrm{~h}$. Protein bands were visualized with ECL reagent (Bio-Rad Laboratories, Inc.) using a gel imager (Thermo Fisher Scientific, Inc.). The gray value of the target protein/internal reference protein was calculated by Image-Pro Plus software (version 6.0; Media Cybernetics, Inc.).

Dual-luciferase reporter assay. The binding sites of circRNA DOCK1 to miR-654-5p and miR-654-5p to SMAD2 were analyzed using the Encyclopedia of RNA Interactomes (ENCORI) database (http://starbase.sysu.edu.cn/index.php). Hep3b cells ( $1 \mathrm{ml}$ cell suspension/well; $5 \times 10^{2}$ cells) seeded into 6-well plates were co-transfected with mimic-NC (50 $\mathrm{nM})$ or miR-654-5p mimic $(50 \mathrm{nM})$ and DOCK1 wild-type (WT)/mutant (MT) $(50 \mathrm{nM})$ or SMAD2 WT/MT $(50 \mathrm{nM})$ with Lipofectamine ${ }^{\circledR} 2000$ (Invitrogen; Thermo Fisher Scientific, Inc.). The mutated 3'-UTR was generated using a site directed mutagenesis kit (Agilent Technologies, Inc.). At $48 \mathrm{~h}$ post-transfection, luciferase activities were measured using a dual-luciferase assay kit (Beijing Solarbio Science \& Technology Co., Ltd.). Renilla luminescence was used as the internal reference.

Statistical analysis. Statistical analyses were performed using SPSS software (version 20.0; IBM Corp). Data are presented as the mean \pm SD of at least three independent experiments. Comparisons among multiple groups were analyzed using one-way ANOVA followed by the Tukey's post hoc test. $\mathrm{P}<0.05$ was considered to indicate a statistically significant difference.

\section{Results}

Interference with circRNA DOCK1 inhibits HCC cell proliferation, invasion and migration. DOCK1 expression in HCC cells was higher compared with that in HHL-5 cells, and DOCK1 expression was the highest in Hep3b cells among the HCC cell lines; therefore, Hep3b cells were selected for the subsequent experiments (Fig. 1A). Following transfection with shRNA-DOCK1\#1/2, DOCK1 expression was decreased. DOCK1 expression in shRNA-DOCK1\#2-transfected Hep3b cells was lower compared with in shRNA-DOCK1\#1 transfected Hep3b cells; therefore, shRNA-DOCK1\#2 was selected for the subsequent experiments (Fig. 1B). DOCK1 knockdown suppressed Hep3b cell activity (Fig. 1C), proliferation (Fig. 1D), migration (Fig. 1E) and invasion (Fig. 1F).

circRNA DOCK1 serves as a sponge of miR-654-5p to negatively regulate miR-654-5p expression. DOCK1 expression was higher in the cytoplasm compared with the nucleus, which was confirmed by cell fractionation and RT-qPCR (Fig. 2A).

Using the ENCORI database, it was predicted that circRNA DOCK1 could bind to miR-654-5p (Fig. 2B). The dual-luciferase reporter assay results indicated that the level of luc/R-Luc in Hep3b cells transfected with DOCK1 WT and miR-654-5p mimic was decreased, which indicated that DOCK1 bound to miR-654-5p (Fig. 2C). miR-654-5p expression in HCC cells was lower compared with HHL-5 cells, and miR-654-5p expression was the lowest in Hep3b cells among the HCC cell lines; therefore, Hep3b cells were selected for subsequent experiments (Fig. 2D). Following transfection with miR-654-5p mimic or miR-654-5p inhibitor, miR-654-5p expression was significantly increased in the miR-654-5p mimic group, but decreased in the miR-654-5p inhibitor group (Fig. 2E). Following transfection with shRNA-DOCK1, miR-654-5p expression was upregulated in Hep3b cells (Fig. 2F).

miR-654-5p directly targets SMAD2. SMAD2 mRNA and protein expression levels in HCC cells were higher compared with HHL-5 cells, and SMAD2 expression was the highest in Hep $3 b$ cells among the HCC cell lines; therefore, Hep3b cells were selected for subsequent experiments (Fig. 3A and B). Using the ENCORI database, it was previously predicted that miR-654-5p could bind to SMAD2 (Fig. 3C). The level of luc/R-Luc in Hep3b cells transfected with SMAD2 WT and miR-654-5p mimic was decreased, which indicated that miR-654-5p bound to SMAD2 (Fig. 3D). Following transfection with miR-654-5p mimic, SMAD2 mRNA and protein expression levels were decreased (Fig. 3E and F). Following transfection with shRNA-DOCK1, SMAD2 mRNA and protein expression levels were decreased (Fig. 3G and H).

HCC cell proliferation, invasion and migration are promoted by the circRNA DOCK1/miR-654-5p/SMAD2 axis. SMAD2 mRNA and protein expression levels in Hep3b cells transfected with pcDNA3.1-SMAD2 were increased (Fig. 4A and B). Interference with DOCK1 decreased Hep3b cell activity (Fig. 4C), proliferation (Fig. 4D), migration (Fig. 4E) and invasion (Fig. 4F), which was partially reversed by miR-654-5p knockdown and SMAD2 overexpression.

\section{Discussion}

HCC represents a serious public health concern worldwide and it is associated with low survival rates (22). To improve the survival rate of patients with $\mathrm{HCC}$, it is important to elucidate the mechanism underlying HCC occurrence and identify novel diagnostic markers and therapeutic targets.

Recent studies demonstrated that circRNAs were implicated in the occurrence and development of HCC, serving an important role in numerous biological processes; therefore, circRNAs may serve as potential diagnostic biomarkers and 

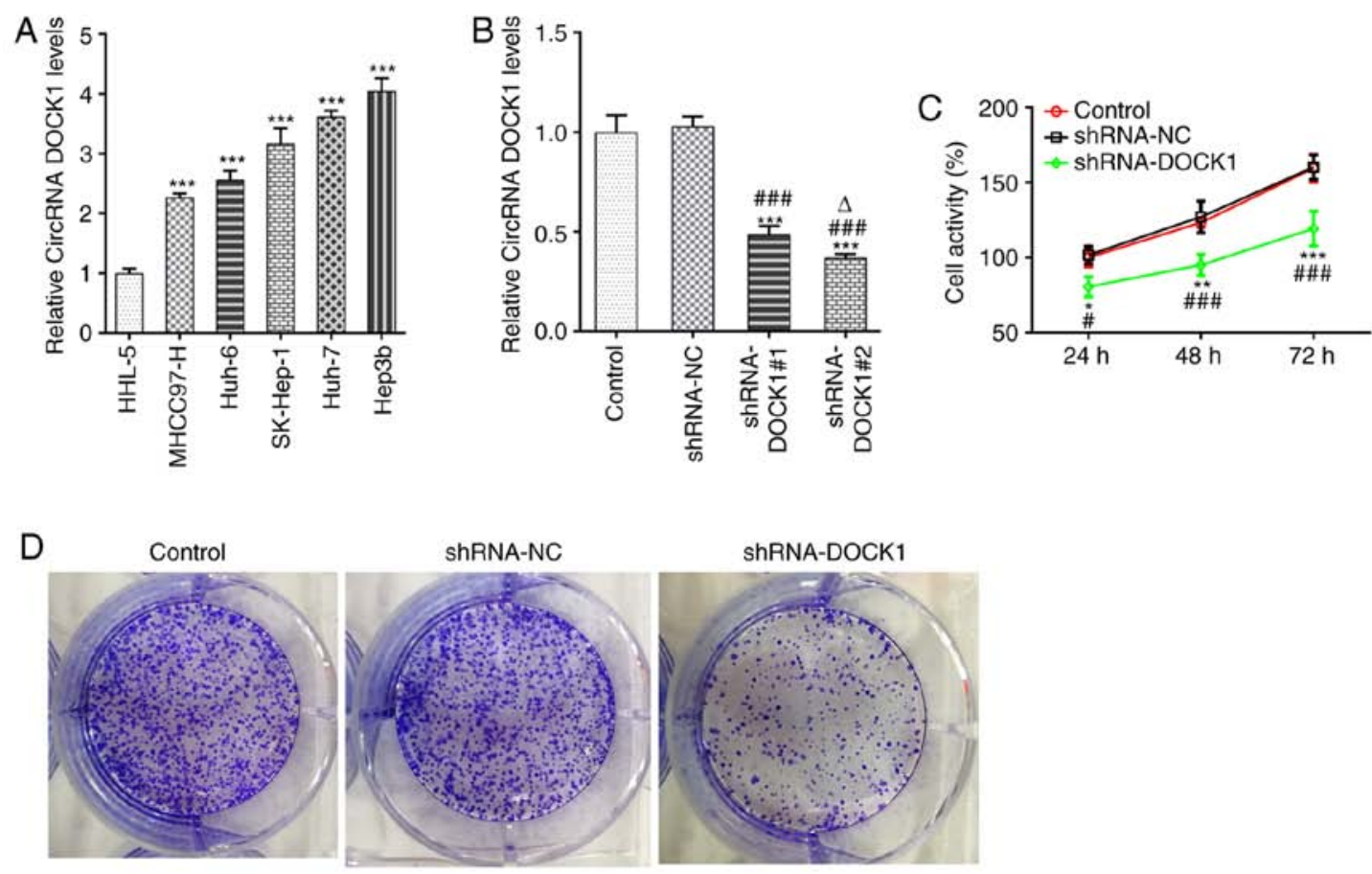

$\mathrm{E}$

Control

ShRNA-NC

ShRNA-DOCK1
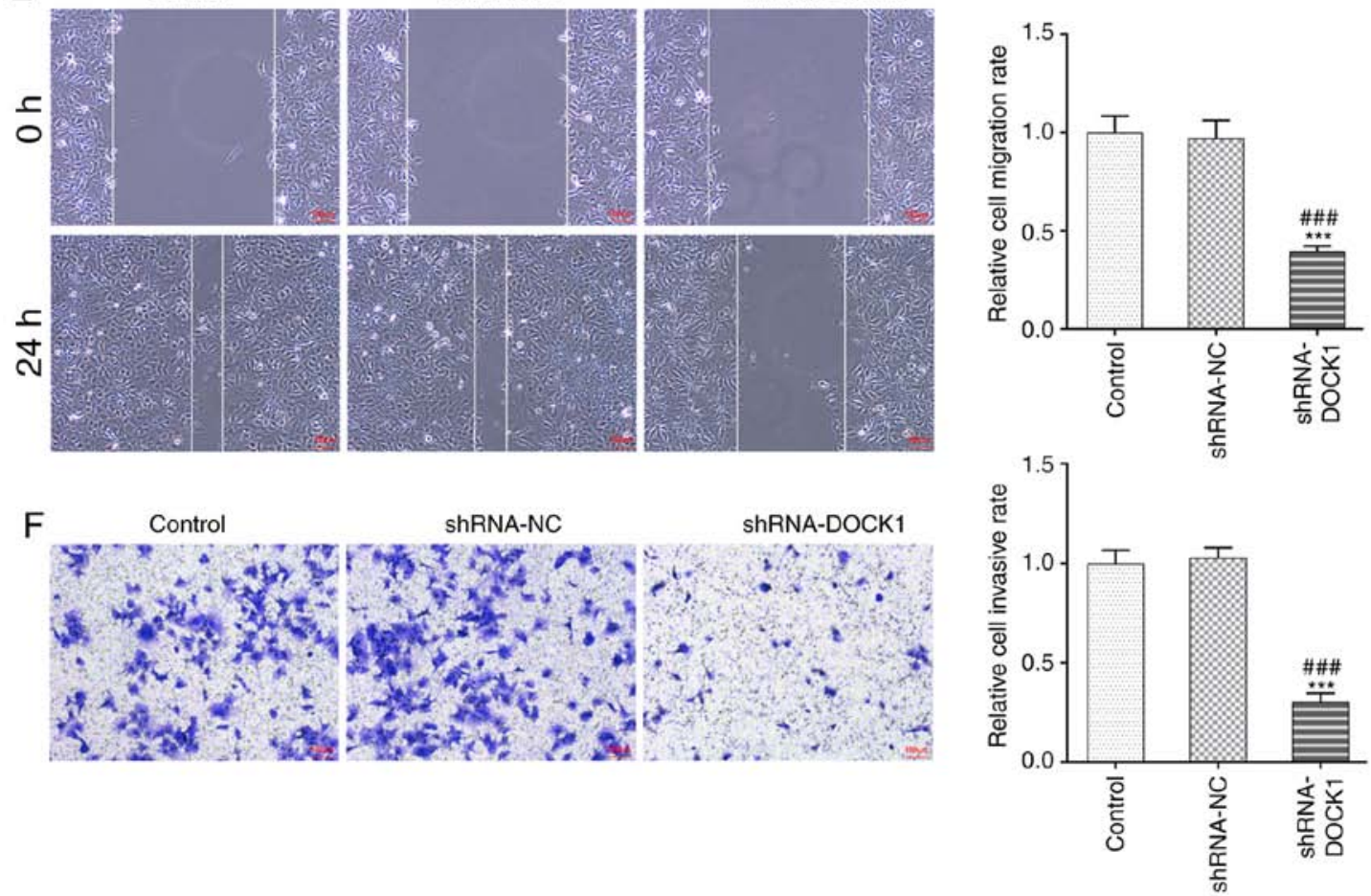

Figure 1. Interference with circRNA DOCK1 inhibits the proliferation, invasion and migration of HCC cells. (A) The circRNA DOCK1 expression in HCC cells was analyzed by RT-qPCR analysis. ${ }^{* * *} \mathrm{P}<0.001$ vs. HHL-5 group. (B) The circRNA DOCK1 expression in HCC cells transfected with shRNA-DOCK1\#1/2 was analyzed by RT-qPCR analysis. ${ }^{* * *} \mathrm{P}<0.001$ vs. Control group; ${ }^{\# \# \#} \mathrm{P}<0.001$ vs. shRNA-NC group; ${ }^{~} \mathrm{P}<0.05$ vs. shRNA-DOCK1\#1 group. (C) The activity of Hep3b cells transfected with shRNA-DOCK1 was detected using a Cell Counting Kit-8 assay. ${ }^{*} \mathrm{P}<0.05,{ }^{* *} \mathrm{P}<0.01$ and ${ }^{* * * *} \mathrm{P}<0.001$ vs. Control group; ${ }^{*} \mathrm{P}<0.05$ and

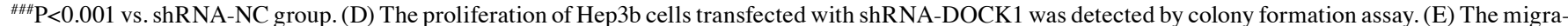
tion of Hep3b cells transfected with shRNA-DOCK1 was detected by wound healing assay. ${ }^{* * *} \mathrm{P}<0.001$ vs. Control group; ${ }^{\# \#} \mathrm{P}<0.001$ vs. shRNA-NC group. (F) The invasion of Hep3b cells transfected with shRNA-DOCK1 was detected by Transwell assay. ${ }^{* * *} \mathrm{P}<0.001$ vs. Control group; ${ }^{\# \# \#} \mathrm{P}<0.001 \mathrm{vs}$. shRNA-NC group. circRNA, circular RNA; DOCK1, dedicator of cytokinesis 1; HCC, hepatocellular carcinoma; RT-qPCR, reverse transcription-quantitative PCR; shRNA, short hairpin RNA; NC, negative control.

therapeutic targets $(23,24)$. A number of circRNAs have been reported to be involved in cancer cell proliferation, invasion and migration. For example, hsa_circ_0005986 was found to be decreased in HCC tissues, and hsa_circ_0005986 reduced the expression levels of target gene Notch1 by binding to miR-129-5p. In addition, hsa_circ_0005986 downregulation 


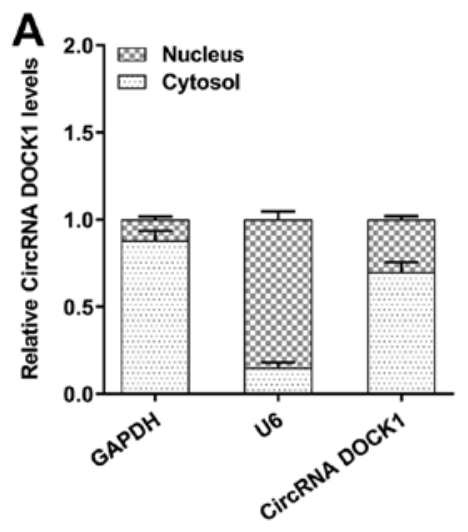

B
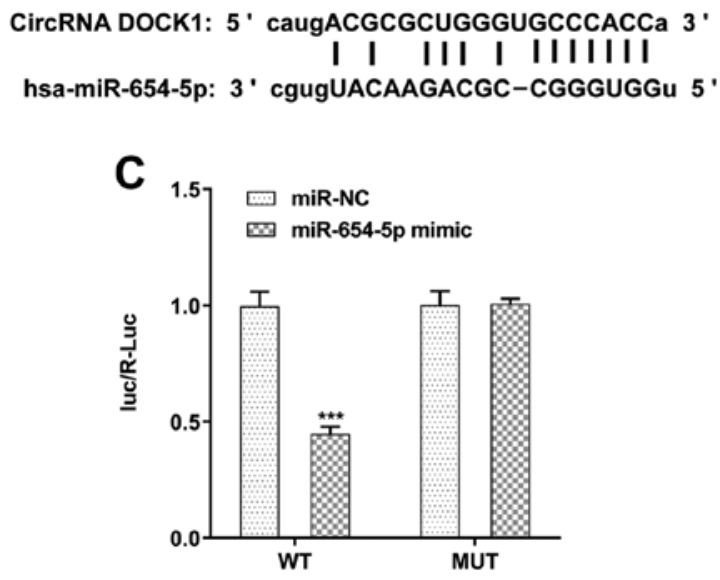

D

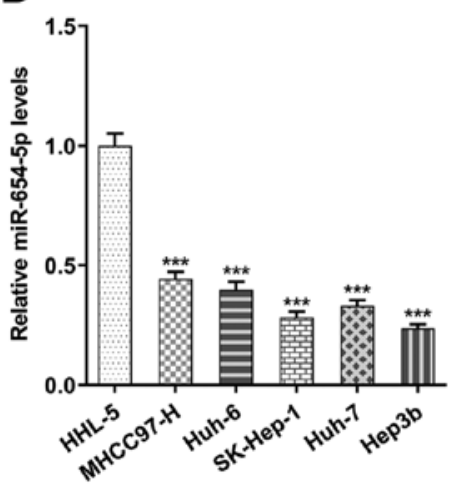

E

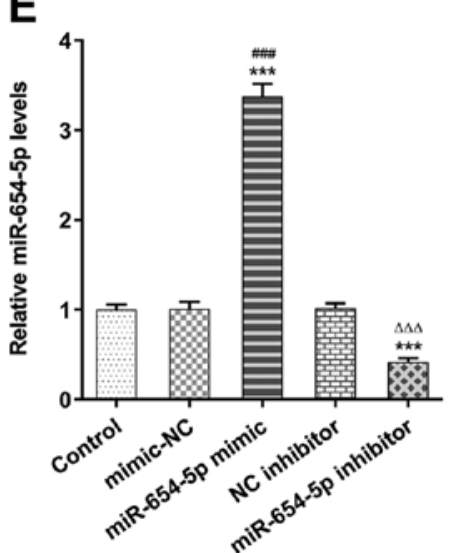

$\mathbf{F}$

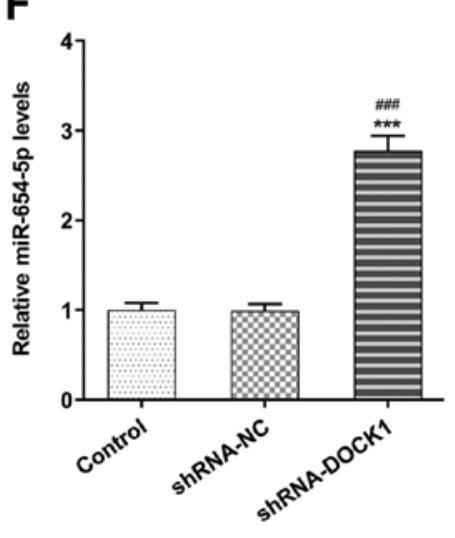

Figure 2. circRNA DOCK1 acts as the sponge of miR-654-5p to negatively regulate the expression of miR-654-5p. (A) The circRNA DOCK1 expression in the cytoplasm and nucleus of Hep3b cells was analyzed by RT-qPCR analysis. (B) The binding sites between circRNA DOCK1 and miR-654-5p. (C) Dual-luciferase reporter assay for confirming the direct binding relationship between circRNA DOCK1 and miR-654-5p. ${ }^{* * *} \mathrm{P}<0.001 \mathrm{vs}$. miR-NC group. (D) The miR-654-5p expression in hepatocellular carcinoma cells was analyzed by RT-qPCR analysis. ${ }^{* * *} \mathrm{P}<0.001$ vs. HHL-5 group. (E) The miR-654-5p expression in Hep3b cells transfected with miR-654-5p mimic/inhibitor was analyzed by RT-qPCR analysis. ${ }^{* * * *} \mathrm{P}<0.001$ vs. Control group; ${ }^{\# \# \#} \mathrm{P}<0.001$ vs. mimic-NC group; ${ }^{\Delta \Delta \Delta} \mathrm{P}<0.001$ vs. NC inhibitor group. (F) The miR-654-5p expression in Hep3b cells transfected with shRNA-DOCK1 was analyzed by RT-qPCR analysis. ${ }^{* * *} \mathrm{P}<0.001$ vs. Control group; ${ }^{\# \# \#} \mathrm{P}<0.001$ vs. shRNA-NC group. circRNA, circular RNA; DOCK1, dedicator of cytokinesis 1 ; miR, microRNA; RT-qPCR, reverse transcription-quantitative PCR; shRNA, short hairpin RNA; NC, negative control; WT, wild-type; MUT, mutant.

promoted HCC cell proliferation via cell cycle transformation (25). Furthermore, $\operatorname{circ} \beta$-catenin was demonstrated to be highly expressed in liver cancer tissues, and knockdown significantly inhibited the malignant phenotype in vivo and in vitro, which activated the Wnt signaling pathway via the internal ribosomal binding site to activate the translation of proteins and promote cancer cell migration (26). Finally, DOCK1 expression has been found to be increased in thyroid cancer, bladder cancer and oral squamous cell carcinoma, and promotes cancer development (11-13). In the present study, it was found that DOCK1 was mainly expressed in the cytoplasm, and the mechanism of competing endogenous RNA takes place in the cytoplasm. In addition, DOCK1 expression was increased in Hep3b cells and the knockdown of DOCK1 suppressed the proliferation, migration and invasion of Hep3b cells, which we speculate could be a potential treatment target of HCC. Therefore, the role of DOCK1 overexpression was not investigated in the present study.

miR-654-3p has been shown to be downregulated in osteosarcoma (OS) tissues and cells, and miR-654-5p overexpression suppressed OS cell proliferation, invasion and migration (27). miR-654-5p was found to be downregulated in breast cancer cells and miR-654-5p overexpression inhibited proliferation and invasion, and promoted apoptosis of breast cancer cells (28). Salt inducible kinase 2 knockdown suppressed the migration and invasion of paclitaxel-resistant ovarian cancer cells, which could be partially reversed by miR-654-5p downregulation (29). In the present study, the results indicated that miR-654-5p directly targeted SMAD2. It was previously reported that inhibition of SMAD2 inhibited the regression of HCC (18-20). In the present study, miR-654-5p expression was decreased and SMAD2 expression was increased in HCC cells. miR-654-5p knockdown or SMAD2 overexpression reversed the inhibitory effects of interference with DOCK1 on HCC cells.

In conclusion, the expression levels of DOCK1 and SMAD2 were increased, and that of miR-654-5p was decreased in HCC cells. Interference with circRNA DOCK1 inhibited HCC cell proliferation, invasion and migration by upregulating miR-654-5p and downregulating SMAD2. In addition, miR-654-5p knockdown or SMAD2 overexpression promoted HCC cell proliferation, invasion and migration. However, 
A

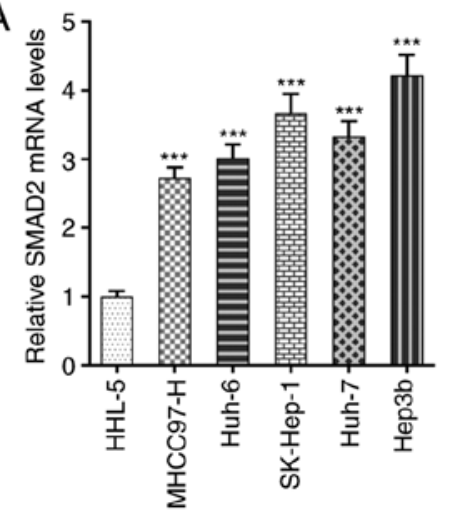

C

SMAD2: 5' acugAUG - UCUUUCUCCCACCu 3'

$$
\text { III III: IIIIII }
$$

hsa-miR-654-5p: 3' cgugUACAAGACGCCGGGUGGu 5'
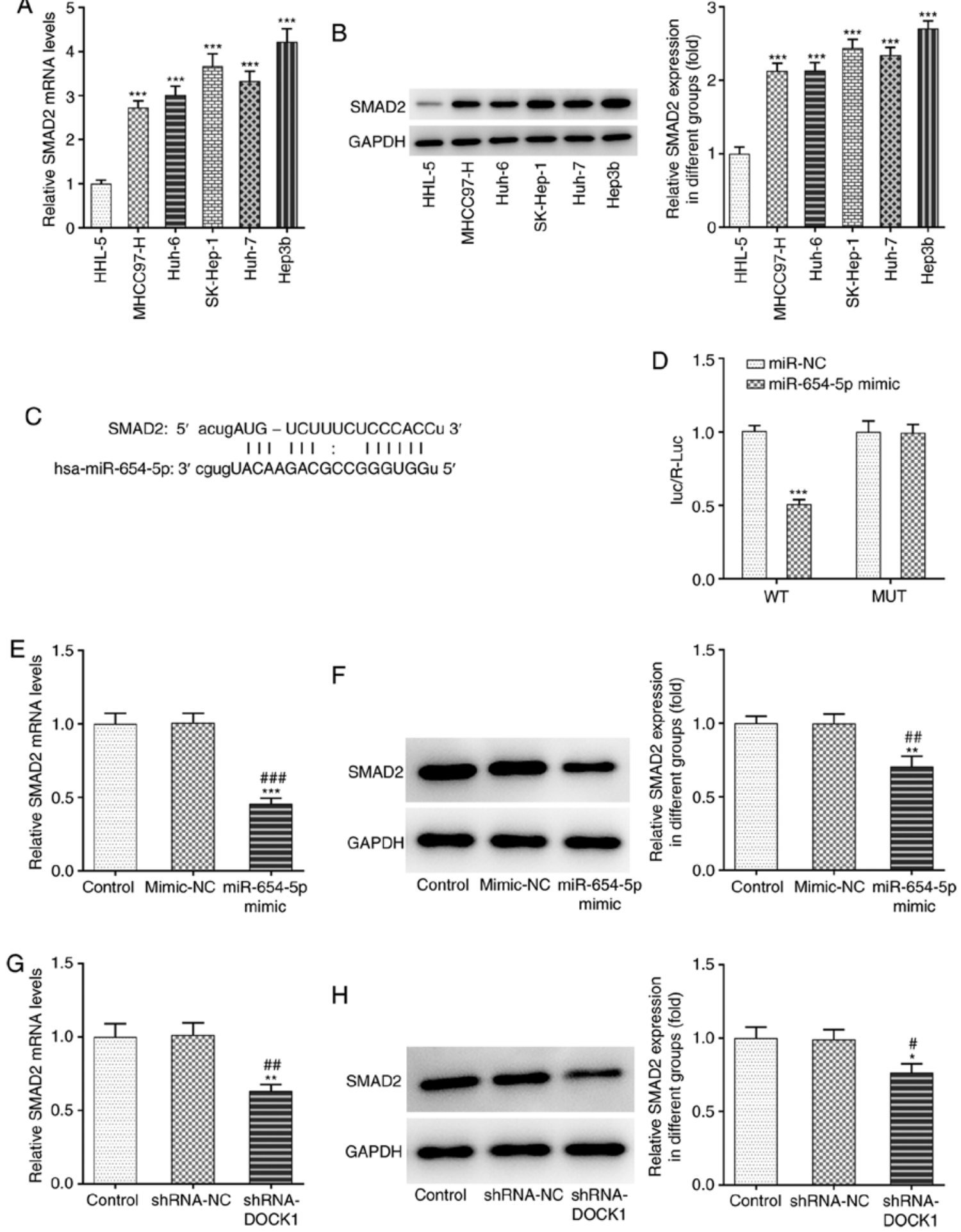

Figure 3. miR-654-5p directly targets SMAD2. (A) The SMAD2 mRNA expression in HCC cells was analyzed by RT-qPCR analysis. (B) The SMAD2 protein expression in HCC cells was determined by western blot analysis. ${ }^{* * *} \mathrm{P}<0.001$ vs. HHL-5 group. (C) The binding sites between miR-654-5p and SMAD2. (D) Dual-luciferase reporter assay for confirming the direct binding relationship between miR-654-5p and SMAD2. ${ }^{* * * *} \mathrm{P}<0.001$ vs. miR-NC group. (E) The SMAD2 mRNA expression in Hep3b cells transfected with miR-654-5p mimic was analyzed by RT-qPCR analysis. (F) The SMAD2 protein expression in Hep3b cells transfected with miR-654-5p mimic was determined by western blot analysis. ${ }^{* *} \mathrm{P}<0.01$ and ${ }^{* * * *} \mathrm{P}<0.001$ vs. Control group; ${ }^{\# \#} \mathrm{P}<0.01$ and ${ }^{\# \# \# P} \mathrm{P}<0.001$ vs. mimic-NC group. (G) The SMAD2 mRNA expression in Hep3b cells transfected with shRNA-DOCK1 was analyzed by RT-qPCR analysis. (H) The SMAD2 protein expression in Hep3b cells transfected with shRNA-DOCK1 was determined by western blot analysis. ${ }^{*} \mathrm{P}<0.05$ and ${ }^{* *} \mathrm{P}<0.01$ vs. Control group; ${ }^{~} \mathrm{P}<0.05$ and ${ }^{\# \#} \mathrm{P}<0.01$ vs. shRNA-NC group. DOCK1, dedicator of cytokinesis 1; miR, microRNA; RT-qPCR, reverse transcription-quantitative PCR; shRNA, short hairpin RNA; NC, negative control; WT, wild-type; MUT, mutant; HCC, hepatocellular carcinoma.

the present study has some limitations. The research would be improved if more cell lines were included for the mechanistic aspect of the study. Since HCC is heterogeneous tumor, it is paramount to investigate multiple in vitro models using different cell lines in order to bring about wholistic changes in the discovery of therapeutic targets. In addition, DOCK1, 


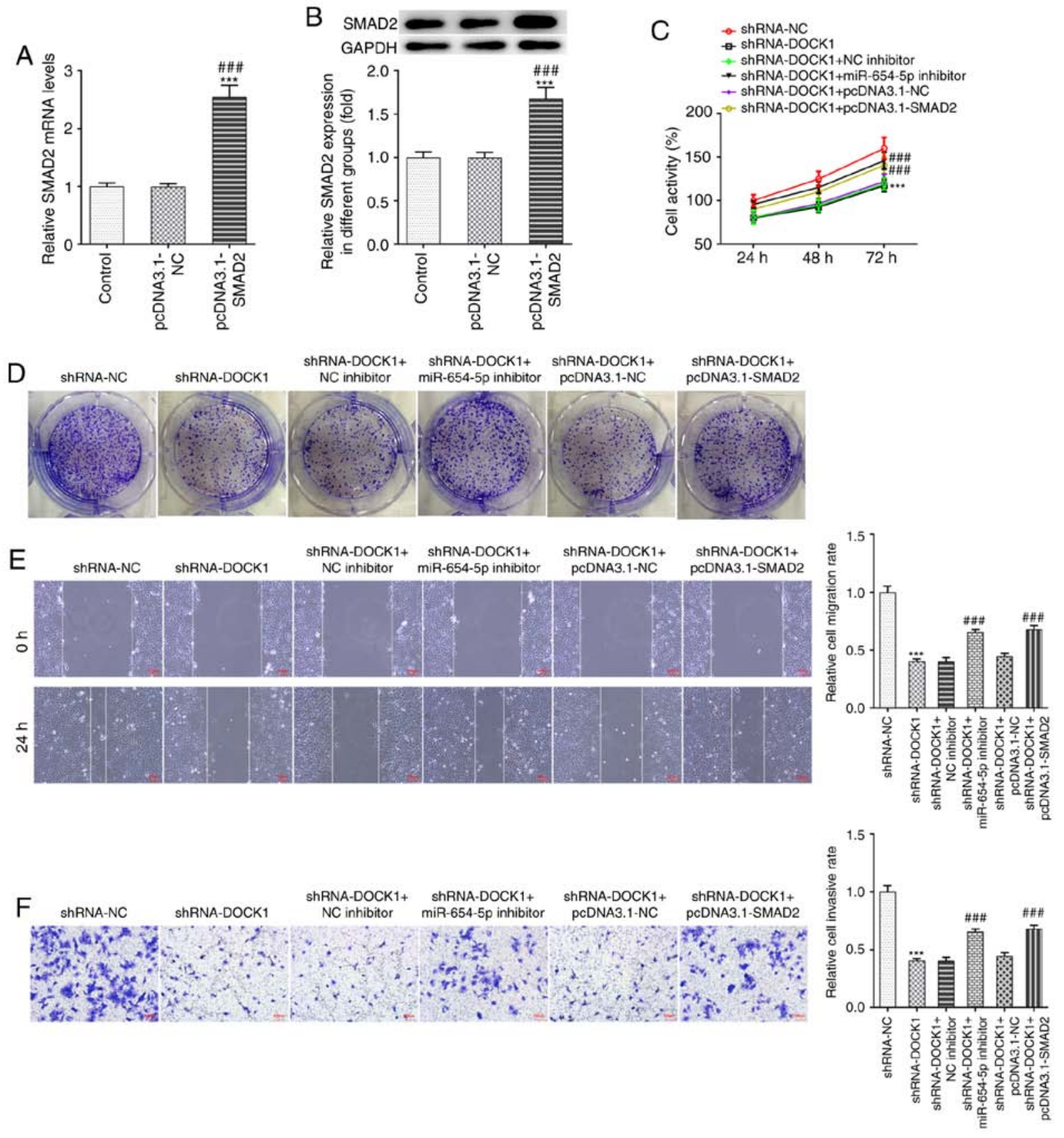

Figure 4. circRNA DOCK1/miR-654-5p/SMAD2 axis promotes proliferation, invasion and migration of HCC cells. (A) The SMAD2 mRNA expression in Hep3b cells transfected with pcDNA3.1-SMAD2 was analyzed by RT-qPCR analysis. (B) The SMAD2 protein expression in Hep3b cells transfected with pcDNA3.1-SMAD2 was determined by western blot analysis. ${ }^{* * * *} \mathrm{P}<0.001$ vs. Control group; ${ }^{* \# \#} \mathrm{P}<0.001$ vs. pcDNA3.1-NC group. (C) The activity of Hep3b cells transfected shRNA-DOCK1 with miR-654-5p inhibitor or pcDNA3.1-SMAD2 was detected using a Cell Counting Kit-8 assay. (D) The proliferation of Hep3b cells transfected shRNA-DOCK1 with miR-654-5p inhibitor or pcDNA3.1-SMAD2 was detected by colony formation assay. (E) The migration of Hep3b cells transfected shRNA-DOCK1 with miR-654-5p inhibitor or pcDNA3.1-SMAD2 was detected by wound healing assay. (F) The invasion of Hep3b cells transfected shRNA-DOCK1 with miR-654-5p inhibitor or pcDNA3.1-SMAD2 was detected by Transwell assay. ${ }^{* * *} \mathrm{P}<0.001$ vs. shRNA-NC group;

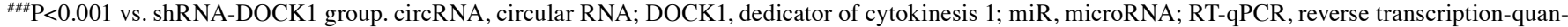
titative PCR; shRNA, short hairpin RNA; NC, negative control; HCC, hepatocellular carcinoma.

miR-654-5p and SMAD2 expression levels in HCC tissues and their association with prognosis of patients with $\mathrm{HCC}$, as well as verification of the molecular mechanisms of these genes in HCC in vivo animal models, should be investigated in the future.

\section{Acknowledgements}

Not applicable.

\section{Funding}

This study was funded by The National Natural Science Foundation of China (grant no. 81572427).

\section{Availability of data and materials}

The datasets used and/or analyzed during the current study are available from the corresponding author on reasonable request. 


\section{Authors' contributions}

YL and JZ acquired the data, confirmed the authenticity of all the raw data, and contributed to the analysis and interpretation of data. YL and YW contributed to the design of the study and drafted the manuscript. All authors read and approved the final manuscript.

\section{Ethics approval and consent to participate}

Not applicable.

\section{Patient consent for publication}

Not applicable.

\section{Competing interests}

The authors declare that they have no competing interests.

\section{References}

1. Yang JD, Hainaut P, Gores GJ, Amadou A, Plymoth A and Roberts LR: A global view of hepatocellular carcinoma: Trends, risk, prevention and management. Nat Rev Gastroenterol Hepatol 16: 589-604, 2019.

2. Llovet JM, Zucman-Rossi J, Pikarsky E, Sangro B, Schwartz M, Sherman M and Gores G: Hepatocellular carcinoma. Nat Rev Dis Primers 2: 16018, 2016.

3. Roayaie S, Jibara G, Tabrizian P, Park JW, Yang J, Yan L, Schwartz M, Han G, Izzo F, Chen M, et al: The role of hepatic resection in the treatment of hepatocellular cancer. Hepatology 62: 440-451, 2015

4. Kulik L and El-Serag HB: Epidemiology and management of hepatocellular carcinoma. Gastroenterology 156: 477-491.e1, 2019.

5. Galle PR, Forner A, Llovet JM, Mazzaferro V, Piscaglia F, Raoul JL, Schirmacher P and Vilgrain V; European Association for the Study of the Liver. Electronic address: easloffice@easloffice.eu; European Association for the Study of the Liver: EASL Clinical Practice Guidelines: Management of hepatocellular carcinoma. J Hepatol 69: 182-236, 2018.

6. Zhu AX, Kudo M, Assenat E, Cattan S, Kang YK, Lim HY, Poon RT, Blanc JF, Vogel A, Chen CL, et al: Effect of everolimus on survival in advanced hepatocellular carcinoma after failure of sorafenib: The EVOLVE-1 randomized clinical trial. JAMA 312: 57-67, 2014.

7. Lopez PM, Villanueva A and Llovet JM: Systematic review: Evidence-based management of hepatocellular carcinoma - an updated analysis of randomized controlled trials. Aliment Pharmacol Ther 23: 1535-1547, 2006.

8. Patop IL, Wüst S and Kadener S: Past, present, and future of circRNAs. EMBO J 38: e100836, 2019.

9. Jeck WR, Sorrentino JA, Wang K, Slevin MK, Burd CE, Liu J, Marzluff WF and Sharpless NE: Circular RNAs are abundant, conserved, and associated with ALU repeats. RNA 19: 141-157, 2013.

10. Han TS, Hur K, Cho HS and Ban HS: Epigenetic associations between lncRNA/circRNA and miRNA in hepatocellular carcinoma. Cancers (Basel) 12: 2622, 2020.

11. Cui W and Xue J: Circular RNA DOCK1 downregulates microRNA-124 to induce the growth of human thyroid cancer cell lines. Biofactors 46: 591-599, 2020.

12. Liu P, Li X, Guo X, Chen J, Li C, Chen M, Liu L, Zhang X and $\mathrm{Zu} \mathrm{X}$ : Circular RNA DOCK1 promotes bladder carcinoma progression via modulating circDOCK1/hsa-miR-132-3p/Sox 5 signalling pathway. Cell Prolif 52: e12614, 2019.
13. Wang L, Wei Y, Yan Y, Wang H, Yang J, Zheng Z, Zha J, Bo P, Tang Y, Guo X, et al: CircDOCK1 suppresses cell apoptosis via inhibition of miR 196a 5p by targeting BIRC3 in OSCC. Oncol Rep 39: 951-966, 2018.

14. Kong R: Circular RNA hsa_circ_0085131 is involved in cisplatiresistance of non-small-cell lung cancer cells by regulating autophagy. Cell Biol Int 44: 1945-1956, 2020.

15. Huang F, Wu X, Wei M, Guo H, Li H, Shao Z, Wu Y and Pu J: miR-654-5p Targets HAX-1 to regulate the nalignancy behaviors of colorectal cancer cells. BioMed Res Int 2020: 4914707, 2020.

16. Majem B, Parrilla A, Jiménez C, Suárez-Cabrera L, Barber M, Marín A, Castellví J, Tamayo G, Moreno-Bueno G, Ponce J, et al: MicroRNA-654-5p suppresses ovarian cancer development impacting on MYC, WNT and AKT pathways. Oncogene 38: 6035-6050, 2019.

17. Zhang JX, He WL, Feng ZH, Chen DL, Gao Y, He Y, Qin K, Zheng ZS, Chen C, Weng HW, et al: A positive feedback loop consisting of $\mathrm{C} 12$ orf $59 / \mathrm{NF}-\kappa \mathrm{B} / \mathrm{CDH} 11$ promotes gastric cancer invasion and metastasis. J Exp Clin Cancer Res 38: 164, 2019.

18. Zheng S, Jia Q, Shen H, Xu X, Ling J, Jing C and Zhang B: Treatment with the herbal formula Songyou Yin inhibits epithelial-mesenchymal transition in hepatocellular carcinoma through downregulation of TGF- $\beta 1$ expression and inhibition of the SMAD2/3 signaling pathway. Oncol Lett 13: 2309-2315, 2017.

19. Zheng X, Gai X, Han S, Moser CD, Hu C, Shire AM, Floyd RA and Roberts LR: The human sulfatase 2 inhibitor 2,4-disulfonylphenyl-tert-butylnitrone (OKN-007) has an antitumor effect in hepatocellular carcinoma mediated via suppression of TGFB1/SMAD2 and Hedgehog/GLI1 signaling. Genes Chromosomes Cancer 52: 225-236, 2013.

20. Wang J, Liu G, Li Q, Wang F, Xie F, Zhai R, Guo Y, Chen T, Zhang N, Ni W, et al: Mucin1 promotes the migration and invasion of hepatocellular carcinoma cells via JNK-mediated phosphorylation of Smad2 at the C-terminal and linker regions. Oncotarget 6: 19264-19278, 2015.

21. Livak KJ and Schmittgen TD: Analysis of relative gene expression data using real-time quantitative PCR and the 2(-Delta Delta C(T)) Method. Methods 25: 402-408, 2001.

22. Forner A, Reig M and Bruix J: Hepatocellular carcinoma. Lancet 391: 1301-1314, 2018.

23. Qin M, Liu G, Huo X, Tao X, Sun X, Ge Z, Yang J, Fan J, Liu L and Qin W: Hsa circ 0001649: A circular RNA and potential novel biomarker for hepatocellular carcinoma. Cancer Biomark 16: 161-169, 2016.

24. Fu L, Wu S, Yao T, Chen Q, Xie Y, Ying S, Chen Z, Xiao B and $\mathrm{Hu}$ Y: Decreased expression of hsa_circ_0003570 in hepatocellular carcinoma and its clinical significance. J Clin Lab Anal 32: e22239, 2018

25. FuL,Chen Q,YaoT,LiT, Ying S,Hu Y andGuoJ:Hsa_circ_0005986 inhibits carcinogenesis by acting as a miR-129-5p sponge and is used as a novel biomarker for hepatocellular carcinoma. Oncotarget 8: 43878-43888, 2017.

26. Liang WC, Wong CW, Liang PP, Shi M, Cao Y, Rao ST, Tsui SK, Waye MM, Zhang Q, Fu WM, et al: Translation of the circular RNA circ $\beta$-catenin promotes liver cancer cell growth through activation of the Wnt pathway. Genome Biol 20: 84, 2019.

27. Xu XZ, Song H, Zhao Y and Zhang L: MiR-654-5p regulated cell progression and tumor growth through targeting SIRT6 in osteosarcoma. Eur Rev Med Pharmacol Sci 24: 3517-3525, 2020.

28. Tan YY, Xu XY, Wang JF,Zhang CW and Zhang SC: MiR-654-5p attenuates breast cancer progression by targeting EPSTI1. Am J Cancer Res 6: 522-532, 2016.

29. Li ZY, Wang XL, Dang Y, Zhu XZ, Zhang YH, Cai BX and Zheng L: Long non-coding RNA UCA1 promotes the progression of paclitaxel resistance in ovarian cancer by regulating the miR-654-5p/SIK2 axis. Eur Rev Med Pharmacol Sci 24: 591-603, 2020.

(i) $($ ) This work is licensed under a Creative Commons Attribution-NonCommercial-NoDerivatives 4.0 International (CC BY-NC-ND 4.0) License. 